\title{
Optimismo disposicional, ansiedad, depresión y estrés en una muestra del Ecuador. Análisis inter-género y de predicción
}

\author{
Dispositional Optimism, Anxiety, Depression and Stress in a \\ Sample of Ecuador. Inter-gender and Prediction Analysis \\ Ismael Gaibor-González ${ }^{1}$ \\ https://orcid.org/0000-0001-9360-3123 \\ Rodrigo Moreta-Herrera ${ }^{2}$ \\ https://orcid.org/0000-0003-0134-5927 \\ ${ }^{1}$ Facultad de Ciencias de la Salud, Universidad Técnica de Ambato, Ecuador \\ ${ }^{2}$ Escuela de Psicología, Pontificia Universidad Católica del Ecuador-Ambato, Ecuador
}

\begin{abstract}
Resumen. Objetivo. Determinar las diferencias inter-género del optimismo disposicional, la depresión, la ansiedad, el estrés y el rol predictor sobre estos rasgos patológicos en una muestra de universitarios del Ecuador. Método. Investigación descriptiva, comparativa, predictora y de corte transversal con las pruebas de Orientación Vital y la Escala de Depresión, Ansiedad y Estrés con 288 participantes de una universidad pública de Ambato, Ecuador (63.9\% son mujeres y 31\% hombres). Con edades entre 17 a 39 años $(M=21.6$ años; $D T=2.5)$. Resultados. Hay diferencias $(p<.05)$ de género en ansiedad y estrés (con mayor presencia en mujeres que en hombres. También, hay correlaciones entre el optimismo disposicional y los rasgos patológicos. Finalmente, el optimismo disposicional explica en la depresión (15.2\%), la ansiedad (9.7\%) y el estrés (5.5\%) los cambios de la varianza. Se concluye que el optimismo disposicional es un predictor de la depresión, la ansiedad y el estrés en estudiantes universitarios.
\end{abstract}

Palabras clave. Ansiedad, depresión, estrés, optimismo, pesimismo.

Abstract. Objective. To determine the inter-gender differences in dispositional optimism, depression, anxiety, and stress; as well as the predictive role of optimism on pathological features in a sample of Ecuadorian university students. Method. This is a descriptive, comparative, predictive and cross-sectional study using the Life Orientation (LOT-R) and Depression, Anxiety and Stress Scale (DASS-21) tests with 288 university students belonging to one university of Ambato; 63.9\% women, 31\% men aged between 17 to $39(M=21.6$ years, $S D=2.5)$. Results. Gender differences were found at the Anxiety and Stress level, with greater presence in women than in men. As regards dispositional optimism and depression, gender is invariant. There were both slight and significant correlations between dispositional optimism and the dimensions evaluated by the DASS 21 test. Dispositional optimism also explains changes in variance in depression (15.2\%), anxiety (9.7\%) and stress (5.5\%). As a conclusion, dispositional optimism is a relevant predictor of depression, as well as anxiety and stress in university students.

Keywords. Anxiety, Depression, Stress, Optimism, Pessimism.

${ }^{1}$ Ismael Gaibor-González. Universidad Técnica de Ambato, Facultad de Ciencias de la Salud. Dirección postal: 180207, Universidad Técnica de Ambato, Facultad de Ciencias de la Salud, Carrera de Psicología Clínica, Ambato, Ecuador. E-mail: ia.gaibor@uta.edu.ec

${ }^{2}$ Rodrigo Moreta-Herrera. Pontificia Universidad Católica del Ecuador, Escuela de Psicología, Ambato, Ecuador. E-mail: rmoreta@pucesa.edu.ec

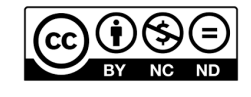

Esta obra está bajo una licencia de Creative Commons Reconocimiento-NoComercial-SinObraDerivada 4.0 Internacional. 


\section{Introducción}

Se entiende por optimismo a la condición o el sentido positivo sobre las expectativas que un individuo se plantea en su futuro próximo; mientras que la situación inversa o negativa se conoce como pesimismo. Esta dualidad optimismo-pesimismo sobre las expectativas a futuro le permiten a las personas anticiparse (aunque no necesariamente de forma racional) a los resultados del futuro próximo; como resultado, asumen tendencias de pensamiento, afecto y conducta, que se mantienen estables ante las diversas situaciones no circunscritas a un aspecto específico de sus vidas (Scheier \& Carver, 1985). Cabe señalar que comprende para una persona un estilo explicativo de las situaciones negativas que afrontan. Las personas pesimistas les atribuyen a las situaciones negativas causas internas, estables y globales; mientras que los optimistas le atribuyen causas externas, inestables y específicas (Lin \& Peterson, 1990).

La postura optimista o pesimista es un estilo de pensamiento anticipatorio, que es aprendido y condicionado y que moldea la conducta. Para algunos autores, esta es una construcción cognitiva de carácter 'situacional' que se relaciona con la motivación y el esfuerzo (Carver \& Scheier, 2014) y que formula las expectativas hacia el futuro; mientras que para otros, es un rasgo de personalidad o 'recurso disposicional' ligado a la satisfacción, el afrontamiento y las respuestas adaptativas a los problemas (Costa-Requena, CantarellAixendri, Parramon-Puig, \& Serón-Micas, 2014), en especial como recurso interpretativo de la realidad negativa (actitudinal). Surge como un mecanismo de autorregulación, que tiene en cuenta el valor del logro que se quiere alcanzar, el compromiso y el esfuerzo necesario (Rasmussen, Wrosch, Scheier, \& Carver, 2006).

El optimismo-pesimismo es abordado teóricamente en distintos modelos y líneas de estudio. Para la Psicología Positiva es un cimiento importante de su desarrollo, pues se la considera como una de las 24 fortalezas personales (Peterson \& Seligman, 2004), en el que se busca conocer la experiencia subjetiva positiva de la conducta, los rasgos individuales positivos y las instituciones que promueven la calidad de vida y previenen las patologías (Seligman \& Csikszentmihalyi, 2014). Como una condición precursora o de predisposición del bienestar general (Forgeard \& Seligman, 2012), el bienestar subjetivo (hedónico) como lo es la satisfacción con la vida y los afectos (Vera, Pávez, \& Silva, 2012); el bienestar psicológico (eudaimónico) (Vera-Villarroel et al., 2016) y el crecimiento postraumático en personas víctimas de desastres naturales, en el que al parecer modifica la percepción de severidad subjetiva del evento vivido (García, Reyes, \& Cova, 2014), entre otros.

También, se analiza el optimismo en el área laboral, por medio del malestar psicológico en el trabajo (Emanuel, Colombo, \& Ghislieri, 2013). En el área educativa se halla que el optimismo es un predictor de la adaptación y la permanencia académica, la cancelación y la repetición de asignaturas (Pérez, 2015). Y en el área de las actividades deportivas como parte del proceso de estrés-recuperación entre nadadores (Aranzana et al., 2016). Finalmente, en el área de la salud, en especial como factor disposicional en la calidad vida, la salud mental y la psicopatología (Quiceno \& Vinaccia, 2014; Cabarcas-Acosta \& Londoño-Pérez, 2014).

Asimismo, interesa este componente en ciertos factores sociodemográficos como el género, en el que aún no queda clara la presencia o no de diferencias, en especial como 
rasgo de personalidad. Así, por ejemplo, Jacobsen, Lee, Marquering y Zhang (2014) encontraron que los hombres son más optimistas que las mujeres ante una amplia gama de problemas, incluidos los de tipo económico y financiero; mientras que Steca et al., (2015); Hinz et al., (2017) concluyeron que este es invariante a nivel de género. En Ecuador, los estudios que analizan el comportamiento del optimismo y el pesimismo son limitados y no se encuentran sistematizados, por lo que abordar investigaciones sobre esta temática, en especial acompañado por el factor salud y enfermedad, resulta ser altamente significativo.

Optimismo y salud

La salud mental requiere de un amplio manejo y entendimiento tanto de la dinámica inherente a la misma como de los elementos que predicen su desempeño, dado que la salud (en especial la mental) y los indicadores bienestar se relacionan significativamente (Moreta-Herrera et al., 2018). Además, porque es un mecanismo necesario para adquirir un estado de plenitud en varios ámbitos de la actividad humana (Organización Mundial de la Salud, 2001).

Cuando la salud mental se ve deteriorada y se presenta la psicopatología, la línea de seguimiento se debe realizar no solo con respecto a la evolución y avance de esta, lo cual es relevante, sino también en los mecanismos de protección, tratamiento y recuperación. Sobre todo considerando el nivel de afectación en el que pueden incurrir ciertos grupos vulnerables ante la patología, entre ellos los niños, los adolescentes y los jóvenes (Lynch \& Clarke, 2006), así como la población universitaria (Mayorga-Lascano \& Moreta-Herrera, 2019). Muchos de ellos, quizás más expuestos a condiciones psicopatológicas como la depresión, la ansiedad, el estrés u otros, que son condiciones con mayor incidencia en la población.

Sin embargo, la comprensión y los mecanismos de contención de la psicopatología aún se encuentran en procesos de identificación básica de la funcionalidad, de los factores de incidencia e incluso de la evaluación propiamente dicha (Moreta-Herrera, López-Calle, Ramos-Ramírez, \& López-Castro, 2018). Un ejemplo de ello es la limitada claridad sobre el papel del género en la psicopatología y sus distintas fases (subclínica, prodrómica y clínica). Por ejemplo, en el caso de la depresión, hay estudios que muestran que el género es invariante (Hernández \& Carrillo, 2010), mientras que en otros casos la predominancia es mayor en las mujeres que en los hombres (Pimentel \& Cova, 2011). Otras patologías, sin embargo, como la ansiedad y el estrés, muestran mayor certeza sobre diferencias intergénero, en el que la tendencia es encontrarse con mayor carga sintomatológica en mujeres que en hombres (Pavez, Mena, \& Vera-Villarroel, 2012; Fernández-González, GonzálezHernández, \& Trianes-Torres, 2015; Jerez-Mendoza \& Oyarzo-Barría, 2015). Indagar en estos aspectos aún se reflejan como a una necesidad imperante para la plena identificación de la psicopatología.

Indudablemente, el papel del optimismo y el pesimismo es fundamental en la dinámica de salud-enfermedad. Por una parte, comprende un precursor relevante para potenciar la salud mental (Quiceno \& Vinaccia, 2014; Vera-Villarroel et al., 2017) y se involucra como un mecanismo de afrontamiento, asociándose a una resistencia más activa y eficaz ante el malestar (Martínez, Reyes del Paso, García, \& Gonzáles, 2006). También, en el establecimiento de una adecuada relación entre el paciente y su cuidador, pues incide en el 
control de la rabia y la ira, así como en el mantenimiento de la salud física del cuidador (López, Romero-Moreno, Márquez-González, \& Losada, 2015). Así mismo, incide en el bienestar mental (Torres-Salazar, Moreta-Herrera, Ramos-Ramírez, \& López-Castro, 2020) y físico, promueve conductas adaptativas, respuestas cognitivas como la capacidad para resolver problemas, flexibilidad mental y procesamiento de la información negativa (Conversano et al., 2010), los hábitos saludables (Ayres, 2008), la percepción del estado de salud y la calidad de vida de las personas (Davis, 2003), la funcionalidad social (Gasquez, Perez, Mercader, \& Inglés, 2014) y como un elemento de apoyo en el afrontamiento al padecimiento de enfermedades físicas y factores asociados, como en el cáncer (Pujol \& Guiteras, 2014).

Por otra parte, la ausencia de optimismo o su contrapolarización hacia el pesimismo, refleja una relación con el número de ingresos hospitalarios, la comorbilidad con enfermedades físicas o mentales, el tiempo de los pacientes en diálisis en personas de edad media (Morales et al., 2011), en el malestar físico en el que parece actuar como factor protector de la experiencia (Remor, Amorós, \& Carrobles, 2006) y en la poca calidad de vida de personas de la tercera edad (Londoño \& Velasco, 2015). Desde el punto de vista de la psicopatología, existe evidencia documentada del optimismo y el pesimismo asociados con enfermedades mentales (Zeb, Riaz, \& Jahangir, 2015).

En esta línea de análisis, la condición optimismo-pesimismo se encuentra involucrada en aspectos específicos de la sintomatología psicológica y en muchos casos, permite la interpretación de estos (Sánchez et al., 2010). Esto se explica por la distorsión de los procesos psíquicos que operan de forma deliberada y automática, generando un desajuste sistémico (Moreta-Herrera \& Reyes-Valenzuela, 2019). Ejemplos que datan de la relación con la psicopatología de forma inversa con la sintomatología depresiva (SánchezHernández \& Méndez, 2009) la ansiógena (que tiene como predictor a la rumiación negativa) (Pimentel \& Cova, 2011); las manifestaciones conductuales y emocionales del estrés (Fernández-González et al., 2015).

En síntesis, lo revisado previamente muestra la necesidad de ampliar los estudios sobre el mecanismo de acción del eje optimismo-pesimismo sobre las condiciones psicopatológicas, en especial en condiciones de mayor prevalencia como el estrés, la ansiedad y la depresión. Lo anterior permitiría formular mecanismos explicativos confirmatorios en grupos sociales poco explorados como lo es la población ecuatoriana, así como el papel predictor para los procesos de intervención a futuro.

A la luz de los aspectos analizados en el presente trabajo se estiman los siguientes objetivos de trabajo: $\mathrm{H}_{1}$ : Determinar la presencia de diferencias por género en el optimismo y la depresión, la ansiedad y el estrés; pues se estima la presencia de dichas diferencias, en el que existe mayor optimismo en hombres que en mujeres; u mayores niveles de depresión, ansiedad y estrés en mujeres que en hombres; $\mathrm{H}_{2}$ : Determinar el grado de relación estadística entre el optimismo en la depresión, la ansiedad y el estrés; puesto que se hipotetiza que existe; una relación inversa entre el optimismo y las variables psicopatológicas y $\mathrm{H}_{3}$ : Identificar si el optimismo es un predictor de las variables psicopatológicas mencionadas, pues se estima que presente una determinación significativa sobre estas. 


\section{Método}

\section{Diseño}

El trabajo comprende un estudio de carácter cuantitativo aplicado a una muestra representativa de estudiantes de psicología, que es descriptivo correlacional y predictor de corte transversal (Montero \& León, 2007) del optimismo disposicional en la ansiedad, el estrés y la depresión.

\section{Participantes}

Este estudio se realizó en una muestra de 288 estudiantes universitarios, comprendido por un $63.9 \%$ de participantes mujeres y un $36.1 \%$ restante son hombres; mismo que se encuentra ajustado a las características sociodemográficas de la población referencial. Las edades comprendidas fluctuaron entre los 17 a los 39 años con una media de $M=21.6$ años; $D T=2.5$. Además, los y las participantes son estudiantes de una universidad pública de la ciudad de Ambato, que se encuentran realizando sus estudios universitarios de grado entre el primer (14.9\%) y el noveno nivel académico de formación en psicología clínica $(9.7 \%)$. Los estudiantes pertenecen a un nivel socioeconómico medio.

La selección de los participantes se realizó a través de un muestreo no probabilístico con criterios de inclusión, los cuáles fueron:

a) Participación voluntaria para formar parte del estudio.

b) Ser estudiante de la carrera de Psicología Clínica de la Universidad Técnica de Ambato.

c) Tener asistencia regular a las clases impartidas en la universidad.

d) Constancia por escrito de consentimiento para difusión de resultados.

\section{Instrumentos}

Escala de depresión, ansiedad y estrés (DASS-21), (Lovibond \& Lovibond, 1995). Instrumento diseñado originalmente en la lengua inglesa para la evaluación independiente de tres categorías psicopatológicas: depresión (he sentido que no había nada que me ilusionara); ansiedad (he tenido temblores); estrés (me ha resultado difícil relajarme) en un cuestionario de 21 ítems. El trabajo actual se desarrolló con la versión al castellano y adaptada para la población española (Ruiz et al., 2017). Las preguntas se califican en una escala de respuesta tipo Likert de 0 a 3 opciones, en el que 0 es 'nada aplicable a mí' y 3 es 'es muy aplicable a mí' o 'aplicable la mayor parte del tiempo'. Este test ha sido validado por estudiantes universitarios chilenos y alcanza una confiabilidad en conjunto de $\alpha=.91 \mathrm{y}$ en las dimensiones de estrés $\alpha=.83$; ansiedad $\alpha=.73$; y depresión de $\alpha=.85$ (Antúnez $\&$ Vinet, 2011) que se interpretan como altamente aceptables. En los participantes del presente estudio, los valores alcanzados fueron a nivel global de $\alpha=.93$ ( $\alpha$ (hombres) $=$ $.926 \mathrm{y} \alpha$ (mujeres) $=.933)$ y en las dimensiones de estrés $\alpha=.82(\alpha$ (hombres) $=.84 \mathrm{y} \alpha$ (mujeres) $=.81)$; ansiedad $\alpha=.86$ ( $\alpha$ (hombres) $=.83 \mathrm{y} \alpha$ (mujeres) $=.86$ ) y en depresión de $\alpha=.85$ ( $\alpha$ (hombres) $=.84 \mathrm{y} \alpha$ (mujeres) $=.85)$.

Test de Orientación de vida (LOT-R), (Scheier, Carver, \& Bridges, 1994). En la versión al castellano y adaptada a población chilena (Ortiz, Gómez-Pérez, Canoino, \& BarreraHerrera, 2016). Esta escala comprende la medición del optimismo disposicional que tienen 
las personas como rasgo de personalidad. El test consta de 10 ítems, cuyas respuestas se ubican en una escala tipo Likert de 0 a 4 opciones, en el que 4 significa 'muy de acuerdo' hasta 0 'muy en desacuerdo'. La prueba cuenta con dos subescalas, la primera es de optimismo (en tiempos difíciles, suelo esperar lo mejor) con los ítems 1, 4, 10 y la segunda de pesimismo (rara vez espero que las cosas salgan a mi manera) con los ítems 3, 7 y 9. Además, estos deben ser corregidos en sentido inverso para obtener la puntuación global de la Orientación Vital. Acerca de la confiabilidad en el estudio referencial, se alcanzó un valor de $\alpha=.72$; mientras que en los participantes ecuatorianos se obtuvo un valor de $\alpha=$ .6 de manera global $(\alpha$ (hombres $)=.42$ y $\alpha$ (mujeres $)=.69)$.

\section{Procedimiento}

Con la respectiva autorización de la institución y con la información sobre el proyecto de investigación y los objetivos que se pretenden alcanzar, todos los participantes completaron el consentimiento informado, incluyendo sus datos sociodemográficos y posteriormente se les aplicó de manera grupal y en sus mismas aulas las escalas de Depresión, Ansiedad y Estrés (DASS-21) y la Escala de Orientación Vital (LOT-R). La aplicación fue realizada por un grupo de dos colaboradores capacitados previamente. Consecutivamente, se depuró la información de la evaluación, se tabularon los datos y se trasladaron a una base informatizada para la gestión pertinente de los mismos. Con ello, se probaron las hipótesis previamente planteadas y se realizó el informe de resultados. Por último, los trabajos de difusión científica fueron puestos previamente en conocimiento del Comité Editorial de la Facultad de Ciencias de la Salud de la Universidad Técnica de Ambato para su envío posterior.

\section{Análisis de datos}

El primer grupo de análisis comprende la comparación por sexo para determinar la existencia o no de diferencias estadísticamente significativas $(p<.05)$ por medio de la prueba tof student $(t)$ asumiendo varianzas iguales (Levene $>0.05)$ o no $($ Levene $<0.05)$ en las variables de trabajo. Posteriormente se realizó el análisis de correlación y el de predicción. El primero se basó en el Coeficiente momento-producto de Pearson $(r)$ entre todas las variables; mientras que posteriormente se realizó el análisis de regresión simple en el que de forma independiente se identificó la determinación del optimismo disposicional con la depresión, la ansiedad y el estrés.

La gestión informática de los casos se realizó a través del software R Commander (R Core Team, 2015) que incluyó los análisis descriptivos y comparativos por género, así como los análisis de correlación y de regresión lineal simple.

\section{Resultados}

\section{Análisis comparativo por género}

Análisis de la Orientación Vital. Con respecto a la evaluación del optimismo disposicional, visto en la Tabla 1, los valores muestran entre los participantes un nivel bajo de pesimismo y elevado de optimismo. De manera general, se observa que la tendencia grupal es a mostrarse disposicionalmente como optimistas. Por otra parte, los análisis de la media reflejan la ausencia de diferencias estadísticamente tanto en los componentes constitutivos del LOT-R como en la escala global. 
Análisis de la depresión, ansiedady el estrés. El análisis comparativo como se puede apreciar en la Tabla 2 muestra la presencia de diferencias estadísticamente significativas por género a nivel de ansiedad $t=-2.24 ; p<.05$ y estrés $t=-2.90 ; p<.01$ en el que las mujeres muestran una mayor puntuación que los hombres, por lo que se puede estimar que ambos grupos son diferentes; además esto se corrobora con la presencia de efecto, cuyos tamaños existentes son pequeños. En el caso de los síntomas depresivos, el aspecto del género resulto ser invariante $(\phi>.05)$. Sobre los puntajes alcanzados, se evidencia que, si bien existen manifestaciones sintomatológicas, estas son considerablemente bajas.

Tabla 1

Análisis comparativo por género en la Orientación Vital

\begin{tabular}{|c|c|c|c|c|c|c|c|}
\hline \multirow[t]{2}{*}{ Variables } & \multicolumn{2}{|c|}{ Hombres } & \multicolumn{2}{|c|}{ Mujeres } & \multirow[t]{2}{*}{$t$} & \multirow[t]{2}{*}{$p$} & \multirow[t]{2}{*}{$g_{(a j .)}$} \\
\hline & $M$ & $D T$ & $M$ & $D T$ & & & \\
\hline Pesimismo & 5.14 & 2.41 & 4.94 & 2.35 & .69 & .505 & .08 \\
\hline Optimismo & 9.29 & 2.01 & 8.83 & 1.92 & 1.91 & .057 & .24 \\
\hline $\begin{array}{c}\text { Orientación } \\
\text { vital }\end{array}$ & 16.15 & 3.21 & 15.89 & 3.46 & .60 & .526 & .08 \\
\hline
\end{tabular}

Tabla 2

Análisis comparativo por género en la Orientación Vital y la depresión, ansiedad y el estrés

\begin{tabular}{|c|c|c|c|c|c|c|c|}
\hline \multirow[t]{2}{*}{ Variables } & \multicolumn{2}{|c|}{ Hombres } & \multicolumn{2}{|c|}{ Mujeres } & \multirow[t]{2}{*}{$t$} & \multirow[t]{2}{*}{$p$} & \multirow{2}{*}{$g_{(a j .)}$} \\
\hline & $M$ & $D T$ & $M$ & $D T$ & & & \\
\hline Depresión & 4.34 & 3.88 & 4.71 & 4.19 & -.74 & .460 & .09 \\
\hline Ansiedad & 3.90 & 3.77 & 5.03 & 4.67 & -2.24 & .026 & .26 \\
\hline Estrés & 6.15 & 4.01 & 7.64 & 4.27 & -2.90 & .004 & .36 \\
\hline
\end{tabular}

Nota. $t=$ Prueba $t$ of student, $p=$ significancia; $g_{(a j .)}=$ Prueba de Hedges Ajustada. 
Análisis de correlaciones

El análisis de correlaciones como se observa en la Tabla 3, muestra que el optimismo disposicional se correlaciona de manera baja y negativa con los indicadores de depresión y ansiedad, pero no así con el estrés. Mientras que, desde el pesimismo, este se correlaciona de igual manera y en las mismas variables, pero de manera positiva.

Por otra parte, si se analiza la composición global de la variable de Orientación Vital, se puede observar que existe un conjunto de correlaciones bajas y negativas con respecto a la depresión, la ansiedad y el estrés. De lo cual, se interpreta que una inclusión de la Orientación Vital (valoración global) positiva repercute en la cantidad e intensidad de los síntomas de depresión, ansiedad y estrés.

Análisis de regresión lineal del optimismo en la depresión, ansiedad y estrés

Se determinó el grado de influencia que tiene el optimismo de manera independiente en cada una de las condiciones patológicas con el Análisis de Regresión Lineal Simple. Este análisis muestra que el optimismo disposicional predice la depresión con una explicación del $15 \%$ de los cambios en la varianza y que representa un buen ajuste como variable predictora $(F=52.59, p<.001)$. Mientras que, en el análisis de la ansiedad, el optimismo disposicional predice los cambios en la varianza en un $10 \%$ con un ajuste significativo ( $F$ $=31.68, p<.001)$. Por último, en los que respecta al Estrés, los hallazgos señalan un poder predictor del $5 \%$ en los cambios de la varianza por parte del optimismo disposicional, el mismo que cuenta con un ajuste pertinente $(F=17.822, p<.01)$.

Tabla 3

Análisis de correlaciones entre la Orientación Vital y la depresión, ansiedad y estrés

\begin{tabular}{lcccccc}
\hline \multicolumn{1}{c}{ Variables } & Optimismo & Pesimismo & LOT-R & Depresión & Ansiedad & Estrés \\
\hline Optimismo & 1 & & & & & \\
Pesimismo & $-.171^{* *}$ & 1 & & & & \\
Orientación vital & $.680^{* *}$ & $-.816^{* *}$ & 1 & & & \\
Depresión & $-.258^{* *}$ & $.337^{* *}$ & $-.391 * *$ & 1 & & \\
Ansiedad & $-.200^{* *}$ & $.238^{* *}$ & $-.277 * *$ & $.684^{* *}$ & 1 & 1 \\
Estrés & $-.169^{* *}$ & $.141^{*}$ & $-.205^{* *}$ & $.686^{* *}$ & $.729 * *$ & 1 \\
\hline$* p<.05 * * p<.01$. & & & & & &
\end{tabular}


Tabla 4

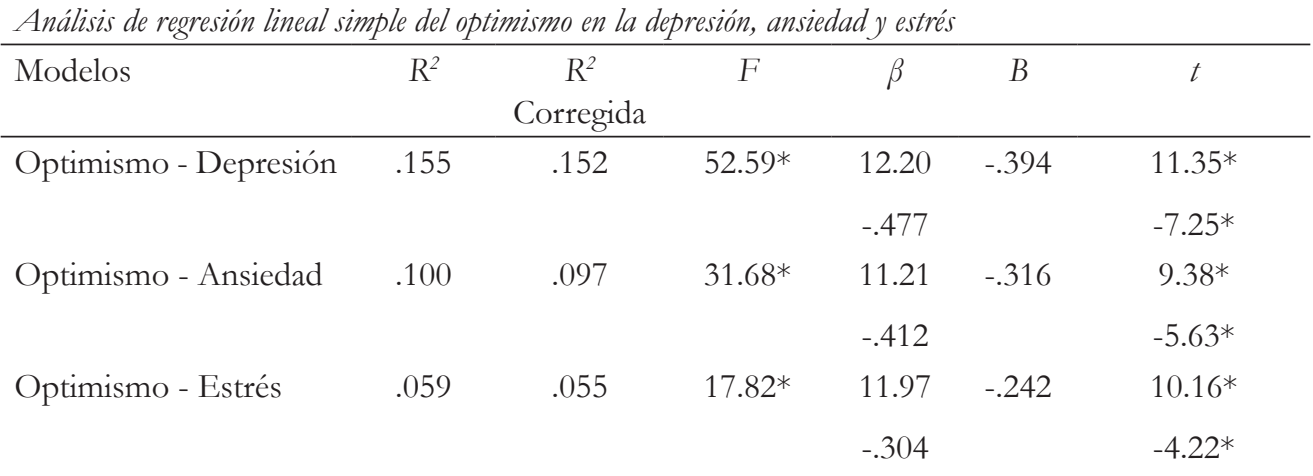

Nota. $R^{2}=$ Coeficiente de Determinación; $F=$ ANOVA; $\beta=$ Coeficiente beta no estandarizado; $B=$ Coeficiente beta estandarizado; $t=$ prueba $\mathrm{t}$.

$* p<.05$

\section{Discusión}

Los objetivos del estudio se centraron en conocer la presencia de diferencias por género en las variables de optimismo disposicional, depresión, ansiedad y estrés en la muestra de participantes del estudio, así como determinar la correlación y determinación del optimismo en la depresión, ansiedad y el estrés.

La evaluación del optimismo disposicional mostró que a nivel de género la presencia de diferencias es inexistente, estableciendo que los grupos muestran un comportamiento similar. Previamente, el estudio de Jacobsen et al. (2014) establecían diferencias por género con predominancia en hombres que, en mujeres, por lo que la hipótesis de diferencias se descarta. Aun así, los hallazgos del estudio son concordantes con otros estudios en los que se concluye que el optimismo es invariante por género (Steca et al., 2015; Hinz et al., 2017). Es decir, que las evidencias arrojan que el género no es relevante desde su estructura social o quizás biológica para la diferenciación del optimismo.

Se encontraron diferencias tanto en la ansiedad como en el estrés. En estos dos casos, las puntuaciones medias fueron significativamente mayores en las mujeres que en los hombres. Los hallazgos actuales confirman la tendencia del género femenino a mostrar mayor sintomatología psicológica que los hombres que previamente fueron detectados en los trabajos de Pavez et al. (2012) y Pimentel y Cova (2011) para la ansiedad; y de Fernández-González et al. (2015) y Jerez-Mendoza y Oyarzo-Barría (2015) para el estrés. En cuanto a las manifestaciones depresivas, los hallazgos señalan la ausencia de diferencias; de este modo hombres y mujeres muestran a la condición sintomatológica como invariante. Este hallazgo corrobora más con lo previsto por Pimentel y Cova (2011) sobre la ausencia de diferencias, que con el trabajo de Hernández y Carrillo (2010). Es probable que estas diferencias se deban a la estructura de la prueba, que no es específica para la depresión como trastorno, sino como de identificación de sintomatología, o a su vez por las características propias de la muestra que en este caso comprenden a estudiantes universitarios. 
De acuerdo con los estudios citados previamente, es claro que el optimismo muestra una correlación con los aspectos de la salud como se preveía por Quiceno y Vinaccia (2014); Vera-Villarroel et al., (2017); y Gasquez et al., (2014), en este caso con los síntomas psicopatológicos y que se confirman en este estudio. Además, que específicamente se relaciona (de forma negativa) con los síntomas de depresión, ansiedad y estrés coincidiendo con los trabajos de Sánchez-Hernández y Méndez (2009); Pavez et al. (2012); FernándezGonzález et al. (2015); y Jerez-Mendoza y Oyarzo-Barría (2015), pero en este caso con población ecuatoriana.

Estos datos, junto con el análisis de regresión, nos llevan a plantear como el eje optimismopesimismo es una entidad casuística de las condiciones psicopatológicas de las personas (al menos en población universitaria). Al considerarse el optimismo disposicional como poco modificable por ser un rasgo de personalidad, el mismo actúa como un escudo protector del surgimiento de patologías o en la disminución de su impacto como lo mencionan Sánchez-Hernández y Méndez (2009). Lo que se observa es que las personas optimistas muestran menos ansiedad y depresión, que los no optimistas, como lo señalan previamente Mustaca, Kamenetzky y Vera (2010). En esta línea de pensamiento, la fuerza predictora que tiene el optimismo sobre la sintomatología es significativa, en especial en la depresión, pues logra explicar el $15.2 \%$ de la varianza; y con menos intensidad en la ansiedad $(9.2 \%)$ y el estrés $(5.5 \%)$. En este aspecto, los estudios que remarcan el poder predictor del optimismo disposicional son escasos, por lo que, desde este punto, el presente trabajo es pionero en ofrecer un mayor avance sobre el entendimiento de las variables estudiadas y en población ecuatoriana, que tradicionalmente es poco estudiada. Además, las implicaciones prácticas de estos avances refuerzan el uso de los procesos de modificación del pensamiento y en este caso de las expectativas de la salud personal como un probable recurso terapéutico para la disminución de la sintomatología con especial énfasis en la depresiva.

El desarrollo del presente trabajo tuvo ciertas limitaciones que los lectores a futuro deben tomar en consideración para la continuidad de estudios similares y nuevas líneas de investigación. Una de las principales limitaciones del estudio, fue el tipo de muestra con la que se trabajó, que en totalidad fueron estudiantes universitarios, por lo que estos resultados no pueden ser generalizados plenamente a otros segmentos poblacionales. Por tanto, motiva a realizar trabajos similares que incluyan adolescentes, población clínica, adultos, entre otros. Así mismo, se trabajó con estudiantes de una universidad pública de la ciudad de Ambato, por lo que se requieren más estudios que incluyan mayor representatividad de la población universitaria.

Otro elemento para considerar es que no se realizaron estudios considerando el factor socioeconómico de los participantes en la interacción con el optimismo y la salud mental; aun cuando existe evidencia de que este elemento tiene una cierta implicación. También, se resalta la limitación que tienen los estudios de correlación, dada la dificultad que se tiene para generar una conclusión de causalidad, debido al fenómeno de circularidad que mantienen este tipo de estudios. Finalmente, un aspecto relevante apunta a que los instrumentos utilizados no cuentan en la actualidad con procesos de validación y adaptación al contexto ecuatoriano. Si bien es cierto, que las pruebas de consistencia interna arrojaron resultados aceptables de 
fiabilidad (excepto en el grupo de hombres en el LOT-R, debido probablemente al número tanto de casos considerados en el estudio como de ítems del factor), es necesario que estos instrumentos y otros similares pasen por pruebas de calibración y validez para que los resultados obtenidos gocen de mayor potencia.

\section{Referencias}

Antúnez, Z., \& Vinet, E. (2011). Escalas de Depresión, Ansiedad y Estrés (DASS 21): Validación de la versión abreviada en estudiantes universitarios chilenos. Terapia Psicologica, 30(3), 49-55. http://dx.doi.org/10.4067/S0718-48082012000300005

Aranzana, M., Salguero, A., Molinero, O., Zubiaur, M., De la Vega, R., Ruíz, R., \& Márquez, S. (2016). Influencia del Perfil Resiliente, Optimismo Disposicional, Estrategias de Afrontamiento y Carga de Entrenamiento en los Niveles de Estrés-Recuperación en Nadadores. Kronos: Revista Universitaria de la Actividad Física y el Deporte, 15(1), 1-12.

Ayres, C.G. (2008). Mediators of the Relationship between Social Support and Positive Health Practices in Middle Adolescents. Journal of Pediatric Health Care, 22(2),94102. https://doi.org/10.1016/j.pedhc.2007.02.010

Cabarcas-Acosta, K. L., \& Londoño-Pérez, C. (2014). Trastornos de la conducta alimentaria, imagen corporal, afrontamiento y optimismo como predictores de aceptación de cirugias plásticas cosméticas. Psicología y Salud, 24(2), 19. https:// doi.org/10.25009/pys.v24i2.925.

Carver, C., \& Scheier, M. (2014). Dispositional optimism. Trends in Cognitive Sciences, 18(6), 293-299. https://doi.org/10.1016/j.tics.2014.02.003.

Conversano, C., Rotondo, A., Lensi, E., Della Vista, O., Arpone, F., \& Reda, M. A. (2010). Optimism and its impact on mental and physical well-being. Clinical practice and epidemiology in mental health, 6, 25-29. http://dx.doi. org/10.2174/1745017901006010025

Costa-Requena, G., Cantarell-Aixendri, M., Parramon-Puig, G., \& Serón-Micas, D. (2014). Optimismo disposicional y estrategias de afrontamiento en pacientes con trasplante renal. Revista de Nefrología, 34(5), 605-611. http://dx.doi.org/10.3265/ Nefrologia.pre2014.Jun.11881

Davis, D.R. (2003). Examining the relationship between optimism, health status, and health behaviors [Tesis doctoral no publicada]. Gonzaga University.

Emanuel, F., Colombo, L., \& Ghislieri, C. (2013). Benessere e malessere emotivo al lavoro nel contesto del call center. Giornale Italiano di Medicina del Lavoro ed Ergonomia, 35(1), A1-A7.

Fernández-González, L., González-Hernández, A., \& Trianes-Torres, M. (2015). Relaciones entre estrés académico, apoyo social, optimismo-pesimismo y autoestima en estudiantes universitarios. Electronic Journal of Research in Educational Psychology, 13(1), 111-130. http://dx.doi.org/10.14204/ejrep.35.14053. 
Forgeard, M., \& Seligman, M. (2012). Seeing the glass half full: A review of the causes and consequences of optimism. Pratiques Psychologiques, 18(2), 107-120.

García, F., Reyes, A., \& Cova, F. (2014). Severidad del trauma, optimismo, crecimiento postraumático y bienestar en sobrevivientes de un desastre natural. Universitas Psychologica, 13(2), 575-584.

Gázquez, J., Pérez, M., Mercader, I., Molero, M., \& Inglés, C. (2014). Repercusión del optimismo y de los Cinco Grandes factores de personalidad sobre la salud de personas mayores. Universitas Psychologica, 13(3). http://dx.doi.org/10.11144/ Javeriana.UPSY13-3.

Hernández, Ó., \& Carrillo, F. (2010). Optimism as a protective factor in child and adolescent depression. Psychology in Spain, 14(1), 42-47.

Hinz, A., Sander, C., Glaesmer, H., Brähler, E., Zenger, M., Hilbert, A., \& Kocalevent, R.(2017). Optimism and pessimism in the general population: Psychometric properties of the Life Orientation Test (LOT-R). International Journal of Clinical and Health Psychology, 17(2), 161-170. https:// doi.org/10.1016/j.ijchp.2017.02.003

Jacobsen, B., Lee, J., Marquering, W., \& Zhang, C. (2014). Gender differences in optimism and asset allocation. Journal of Economic Behavior \& Organization, 107, 630-651. https://doi.org/10.1016/j.jebo.2014.03.007

Jerez-Mendoza, M., \& Oyarzo-Barría, C. (2015). Estrés académico en estudiantes del Departamento de Salud de la Universidad de Los Lagos Osorno. Revista cbilena de neuropsiquiatría, 53(3), 149-157. http://dx.doi.org/10.4067/S0717-92272015000300002

Lin, E., \& Peterson, C. (1990). Pessimistic explanatory style and response to illness. Behaviour Research and Therapy, 28(3), 243-248. https://doi.org/10.1016/00057967(90)90007-6

Londoño, C., \& Velasco, M. (2015). Estilo de afrontamiento, optimismo disposicional, depresión, imagen corporal, IMC y riesgo de TCA como predictores de calidad de vida relacionada con la salud. Psychologia. Avances de la disciplina, 9(2), 35-47.

López, J., Romero-Moreno, R., Márquez-González, M., \& Losada, A. (2015). Anger and health in dementia caregivers: exploring the mediation effect of optimism. Stress and Health, 31(2), 158-165. https://doi.org/10.1002/smi.2539

Lovibond, S., \& Lovibond, P. (1995). Manual for the depression anxiety stress scales. Australia: Psychology Foundation of Australia.

Lynch, F., \& Clarke, G. (2006). Estimating the economic burden of depression in children and adolescents. American journal of preventive medicine, 31(6), 143-151. https://doi. org/10.1016/j.amepre.2006.07.001

Martínez, A., Reyes del Paso, G., García, A., \& Gonzáles, M. (2006). Optimismo/pesimismo disposicional y estrategias de afrontamiento del estrés. Psicothema, 18, 66-72.

Mayorga-Lascano, M., \& Moreta-Herrera, R. (2019). Síntomas clínicos, subclínicos y necesidades de atencion psicológica en estudiantes universitarios con bajo 
rendimiento/Psychological Care Needs for Underperforming College Students with Clinical and Subclinical Symptoms. Educación, 43(2). http://dx.doi. org/10.15517/revedu.v43i2.32239

Montero, I., \& León, O. (2007). A guide for naming research studies in Psychology. International Journal of Clinical and Health Psychology, 7(3), 847-862.

Morales, A., Arenas, M., Reig, A., Álvarez, F., Malek, T., Moledous, A., . . Cotilla, E. (2011). Optimismo disposicional en pacientes en hemodiálisis y su influencia en el curso de la enfermedad. Nefrología (Madrid), 31(2), 199-205. 10.3265/Nefrologia. pre2011.Feb.10534

Moreta-Herrera, R., \& Reyes-Valenzuela, C. (2019). Cognición implícita en psicopatología: contribuciones en el abordaje de los trastornos por consumo de sustancias. Drugs and Addictive Behavior, 4(1), 173-177. https://doi.org/10.21501/24631779.3142

Moreta-Herrera, R., López-Calle, C., Gordón-Villalva, P., Ortíz-Ochoa, W., \& GaiborGonzález, I. (2018). Satisfacción con la vida, Bienestar Psicológico y Social como predictores de la salud mental en ecuatorianos. Actualidades en Psicología, 32(124). http://dx.doi.org/10.15517/ap.v32i124.31989

Moreta-Herrera, R., López-Calle, C., Ramos-Ramírez, M., \& López-Castro, J. (2018). Estructura factorial y fiabilidad del Cuestionario de Salud General de Goldberg (GHQ-12) en universitarios ecuatorianos. Revista Argentina de Ciencias del Comportamiento, 10(3), 35-42. https://doi.org/10.32348/1852.4206. v10.n3.20405

Mustaca, A.E., Kamenetzky, G.V., \& Vera, P. (2010) Relaciones entre variables positivas y negativas en una muestra de estudiantes argentinos. Revista Argentina de Clínica Psicológica, 9(3), 227-235.

Organización Mundial de la Salud. (2001). Fortaleciendo la prevención de salud mental [OMS]. Organización Mundial de la Salud.

Ortiz, M., Gómez-Pérez, D., Canoino, M., \& Barrera-Herrera, A. (2016). Validación de la versión en Español de la Escala de Optimismo Disposicional (LOT-R) en una muestra Chilena de estudiantes universitarios. Terapia psicológica, 34(1), 53-58. http://dx.doi.org/10.4067/S0718-48082016000100006

Pavez, P., Mena, L., \& Vera-Villarroel, P. (2012). El rol de la felicidad y el optimismo como factor protector de la ansiedad. Universitas Psychologica, 11(2), 369-380. https:/ / doi. org/10.11144/Javeriana.upsy11-2.rfof.

Pérez, C. (2015). Optimismo y salud positiva como predictores de la adaptación a la vida universitaria. Acta colombiana de psicología, 12(1), 95-107.

Peterson, C., \& Seligman, M. (2004). Character Strenghts and Virtues: A bandbook and classification. APA \& Oxford Univesity Press.

Pimentel, M., \& Cova, F. (2011). Efectos de la Rumiación y la Preocupación en el Desarrollo de Sintomatología Depresiva y Ansiosa en Estudiantes Universitarios 
de la Ciudad de Concepción, Chile. Terapia psicológica, 29(1), 43-52. http://dx.doi. org/10.4067/S0718-48082011000100005

Pujol, S., \& Guiteras, A. (2014). Optimismo disposicional y calidad de vida en mujeres con cáncer de mama. Psicooncología, 11(1), 19-29. https://doi.org/10.5209/rev_ PSIC.2014.v11.n1.44914

Quiceno, J., \& Vinaccia, S. (2014). Calidad de vida en adolescentes: Análisis desde las fortalezas personales y las emociones negativas. Terapia psicológica, 32(3), 185-200. http://dx.doi.org/10.4067/S0718-48082014000300002

R Core Team. (2015). R: A language and environment for statistical computing. Vienna, Austria: $\mathrm{R}$ Foundation for Statistical Computing.

Rasmussen, H., Wrosch, C., Scheier, M., \& Carver, C. (2006). Self-regulation processes and health: the importance of optimism and goal adjustment. Journal of Personality, 74(6), 1721-1747. https://doi.org/10.1111/j.1467-6494.2006.00426.x

Remor, E., Amorós, M., \& Carrobles, J. (2006). El optimismo y la experiencia de ira en relación con el malestar físico. Anales de Psicología, 22(1), 37-44.

Ruiz, F., García, M., Suárez, J., Falcón., \& Odriozola, P. (2017). El factor de estructura jerárquica de la versión española de la depresión ansiedad y el estrés-21 Escala. International journal of psychology and psychological therapy, 17(1), 97-105.

Sánchez, Ó., Martín, R., Méndez, F., Corbalán, F., \& Limiñana, R. (2010). Relación entre optimismo, creatividad y síntomas psicopatológicos, en estudiantes universitarios. Electronic Journal of Research in Educational Psychology, 8(22), 1151-1178. http:// dx.doi.org/10.25115/ejrep.v8i22.1418

Sánchez-Hernández, Ó., \& Méndez, F. (2009). Optimism as a Protective Factor in Child and Adolescent Depression. Clínica y Salud, 20(3), 273-280.

Scheier, M., \& Carver, C. (1985). Optimism, Coping, and Health: Assessment and Implications of Generalized Outcome Expectancies. Health Psychology, 4(3), 219247. https://doi.org/10.1037/0278-6133.4.3.219

Scheier, M., Carver, C., \& Bridges, M. (1994). Distinguishing optimism from neuroticism (and trait anxiety, self-mastery, and self-esteem): A reevaluation of the Life Orientation Test. Journal of Personality and Social Psychology, 67(6), 1063-1078. https://doi.org/10.1037/0022-3514.67.6.1063

Seligman, M., \& Csikszentmihalyi, M. (2014). Positive psychology: An introduction. En M. Csikszentmihalyi (Ed.), Flow and the foundations of positive psychology (pp. 279298). Netherlands: Springer

Steca, P., Monzani, D., Greco, A., Chiesi, F., \& Primi, C. (2015). Item response theory analysis of the life orientation test-revised: age and gender differential item functioning analyses. Assessment, 22(3), 341-350. https://doi.org/10.1177/1073191114544471

Torres-Salazar, C., Moreta-Herrera, R., Ramos-Ramírez, M., \& López-Castro, J. (2020). Sesgos Cognitivo de Optimismo y Percepción de Bienestar en una Muestra de 
Universitarios Ecuatorianos. Revista Colombiana de Psicología, 29(1), 61-72. https:// doi.org/10.15446/rcp.v29n1.75853

Vera, P., Pávez, P., \& Silva, J. (2012). El Rol Predisponente del Optimismo: Hacia un Modelo Etiológico del Bienestar. Terapia psicológica, 30(2), 77-84. http://dx.doi. org/10.4067/S0718-48082012000200008

Vera-Villarroel, P., Celis-Atenas, K., Urzúa, A., Contreras, D., \& Lillo, S. (2016). Los afectos como mediadores de la relación optimismo y bienestar. Revista Argentina de Clínica Psicológica, 25(2), 195-202.

Vera-Villarroel, P., Urzúa, A., Beyle, C., Contreras, D., Lillo, S., Oyarzo, F., \& Sanín-Posada, A. (2017). Relación entre optimismo y salud bajo la influencia de las creencias religiosas en dos muestras latinoamericanas. Revista Latinoamericana de Psicología, 49(3), 213-221. https://doi.org/10.1016/j.rlp.2017.03.002

Zeb, R., Riaz, M., \& Jahangir, F. (2015). Religiosity and Optimism as Predictors of Psychopathology and Mental Health. Journal of Asian Development Studies, 4(1), 6775. https://doi.org/10.1080/13674670601166505 